\title{
Análise da Validade e Precisão de Instrumento de Diferencial Semântico
}

\section{Analysis of Validity and Precision of a Semantic Differential Instrument}

\author{
João Henrique de Almeida*, $a$, Renato Bortoloti ${ }^{a}$, Paulo Roberto dos Santos Ferreira ${ }^{b}$, \\ Patricia Waltz Schelini ${ }^{a} \&$ Julio César Coelho de Rose ${ }^{a}$ \\ ${ }^{a}$ Universidade Federal de São Carlos, São Carlos, São Paulo, Brasil \\ $\&{ }^{b}$ Universidade Federal da Grande Dourados, Dourados, Mato Grosso do Sul, Brasil
}

\begin{abstract}
Resumo
Este estudo investigou evidências de validade e precisão de um instrumento de diferencial semântico que tem sido utilizado para medir a transferência de significados entre estímulos equivalentes. Um total de 196 estudantes universitários avaliou fotografias de faces expressando emoções e também figuras abstratas, utilizando um instrumento composto por 13 escalas bipolares ancoradas por adjetivos opostos. A análise fatorial dos dados coletados forneceu indicações da validade do instrumento e os coeficientes alfa obtidos indicam que os dois fatores extraídos nesta análise possuem níveis de precisão aceitáveis, embora um dos fatores deva ser revisto. Discute-se que o modelo de equivalência de estímulos, entendido como um modelo experimental de relações simbólicas, pode se beneficiar de instrumentos capazes de fornecer medidas válidas de transferência de significado.

Palavras-chave: Diferencial semântico, validação de medida de significado, equivalência de estímulos, comportamento simbólico.
\end{abstract}

\begin{abstract}
This study assessed evidence of validity and precision of a semantic differential test that has been used to measure the transfer of meaning among equivalent stimuli. Participants were 196 college students who evaluated pictures of faces expressing emotions and also abstract pictures using an instrument comprised by 13 bipolar scales anchored by opposite adjectives. The factor analysis of the data evidenced the validity of the instrument. Values of alpha coefficients indicated that the factors extracted by this analysis have acceptable levels of precision, although one of the factors might be revised. It is discussed that the stimulus equivalence paradigm, an experimental model for symbolic relations, can benefit from instruments that provide valid measures of meaning transfer.

Keywords: Semantic differential, validation of a measure of meaning, stimulus equivalence, symbolic behavior.
\end{abstract}

O modelo de equivalência de estímulos desenvolvido por Sidman e colaboradores (Sidman, 1994; Sidman \& Tailby, 1982) fornece critérios operacionais para identificar funções simbólicas em comportamentos observáveis e para simular em laboratório relações simbólicas que ocorrem

\footnotetext{
* Endereço para correspondência: Centro de Educação e Ciências Humanas, Universidade Federal de São Carlos, Rodovia Washington Luiz, Km 235, Caixa Postal 676, Jardim Guanabara, São Carlos, SP, Brasil 13565-905. E-mail: emaildojoaoh@yahoo.com.br

Esta pesquisa foi realizada como parte das atividades do Instituto Nacional de Ciência e Tecnologia sobre Comportamento, Cognição e Ensino, com financiamento do Conselho Nacional de Desenvolvimento Científico e Tecnológico (CNPq; Processo 573972/2008-7) e Fundação de Amparo à Pesquisa do Estado de São Paulo (FAPESP; Processo 2008/57705-8). O primeiro autor é bolsista de doutorado da FAPESP. O segundo autor contou com auxílio da FAPESP (Jovem Pesquisador, Processo 2011/50561-3) e do CNPq. O último autor é bolsista de produtividade em pesquisa do $\mathrm{CNPq}$
}

naturalmente. O modelo estabelece uma distinção entre relações de pares de estímulos associados (i.e., relações condicionais entre estímulos que seriam desprovidas de função simbólica) e relações simbólicas, chamadas de relações de equivalência. Sidman e Tailby (1982) argumentaram que relações simbólicas podem ser identificadas por meio de testes que revelam indicadores comportamentais derivados de propriedades que não estão presentes em relações entre pares associados.

As pesquisas sobre equivalência de estímulos envolvem pelo menos duas fases, ambas baseadas em procedimentos de emparelhamento ao modelo. Na primeira fase, o participante aprende um conjunto de discriminações condicionais arbitrárias entre estímulos diferentes. Na fase seguinte, verifica-se a emergência de discriminações condicionais (isto é, o estabelecimento de relações condicionais que não foram ensinadas diretamente, mas que podem ser derivadas das relações ensinadas) que comprovam que os estímulos envolvidos são substituíveis entre si. Os 
Almeida, J. H., Bortoloti, R., Ferreira, P. R. S., Schelini, P. W. \& Rose, J. C. C. (2014). Análise das Propriedades Psicométricas de Instrumento de Diferencial Semântico.

trabalhos iniciais de Sidman (1971) e Sidman e Cresson (1973) ilustram bem esse processo. Sidman (1971) trabalhou com um jovem que já sabia relacionar palavras faladas (A) a figuras representativas dessas palavras (B) e ensinou a ele relações entre as mesmas palavras faladas (A) e as palavras impressas correspondentes (C). Quando as relações ensinadas estavam bem estabelecidas, Sidman conduziu uma série de tentativas de emparelhamento ao modelo para testar o desempenho do jovem diante de palavras impressas apresentadas como estímulo modelo e figuras apresentadas como opções de escolha (estímulos de comparação) e também diante de figuras apresentadas como modelo e palavras impressas apresentadas como estímulos de comparação. Essas tarefas revelaram que o participante, que não tinha qualquer habilidade de leitura antes do experimento, mostrou-se então capaz de relacionar as figuras às palavras impressas $(\mathrm{BC})$ e as palavras impressas às figuras (CB), mesmo sem nunca ter sido explicitamente ensinado a fazê-lo. As relações entre palavras impressas e figuras, portanto, emergiram a partir das relações que foram ensinadas no experimento e daquelas que ele já era capaz de estabelecer. Sidman (1971) observou também que, quando cada palavra impressa era apresentada sozinha, o jovem era capaz de dizer o nome dela. Sidman concluiu que as palavras faladas, as figuras e as palavras impressas haviam se tornado estímulos equivalentes e que, em função disso, o jovem podia ler e entendia o que estava lendo. Sidman e Cresson (1973) obtiveram resultados semelhantes com participantes com comprometimento intelectual ainda maior, diagnosticados como severamente retardados.

Posteriormente, Sidman e Tailby (1982) observaram que o termo equivalência tem uma definição rigorosa na matemática, aplicando-se a relações que possuem as propriedades de reflexividade, simetria, e transitividade. Quando aplicado à escolha consistente de, por exemplo, palavras impressas diante de palavras faladas, o termo equivalência estaria assumindo algo além do desempenho observado de escolha dos estímulos de comparação em presença dos respectivos modelos. Esse termo implicaria também a reflexividade, a simetria e a transitividade dessa relação e, portanto, deveria ser usado apenas quando essas propriedades são empiricamente demonstradas. As relações diretamente observadas, segundo Sidman e Tailby (1982), são discriminações condicionais, em que a escolha de cada estímulo de comparação é condicional à apresentação do respectivo modelo. Para demonstrar que as relações condicionais entre estímulos são também relações de equivalência é necessário demonstrar que elas possuem as propriedades de reflexividade, simetria e transitividade. A propriedade reflexiva pode ser testada por meio da escolha de um estímulo de comparação idêntico ao estímulo modelo apresentado (se A1 é apresentado como modelo e A1, A2 e A3 são apresentados como estímulos de comparação, o participante deve selecionar A1). Testes de simetria podem ser feitos pela inversão da ordem de uma relação estabelecida (por exemplo, se o participante escolhe o estímulo de comparação B1 na presença do modelo A1 e B2 na presença do modelo A2, deve escolher o estímulo de comparação A1 na presença do modelo B1 e A2 na presença do modelo B2). A transitividade é determinada pela demonstração de que dois pares de estímulos com um elemento comum estabelecem um novo par (por exemplo, se o participante estabeleceu a relação entre A e B e também entre B e C, deve estabelecer a relação entre A e C). Simetria e transitividade podem ser demonstradas conjuntamente (por exemplo, pelo teste da relação CA depois de ter estabelecido as relações AB e BC; Sidman, 1990; Sidman \& Tailby, 1982). Testes que combinam simetria e transitividade são algumas vezes chamados de testes de equivalência porque evidenciam, simultaneamente, a emergência de duas propriedades das relações de equivalência. A terceira propriedade (reflexiva) é muitas vezes assumida em algumas situações experimentais (e.g., Bortoloti \& de Rose, 2009).

Sidman e colaboradores (e.g., Sidman, 1986, 1994; Sidman \& Tailby, 1982) argumentaram que as relações de equivalência constituem relações com significado semântico. Quando um conjunto de estímulos relacionados entre si constitui uma classe de estímulos equivalentes, cada membro da classe constitui um símbolo dos demais e os membros da classe compartilham o significado. Bortoloti e de Rose (2007) propuseram que uma forma de conferir a validade dessa inferência seria verificar a possibilidade de transferência de significados entre estímulos equivalentes por meio da utilização de alguma técnica de medida externa ao modelo de equivalência. Empregaram, para essa finalidade, a técnica do diferencial semântico introduzida por Osgood e colaboradores (Osgood, Suci, \& Tannenbaum, 1957). O diferencial semântico é uma técnica usada para medir o significado que os participantes atribuem a "conceitos” (palavras, locuções, fotografias, desenhos, etc.) Para tanto é utilizado um instrumento que permite registrar, quantificar e comparar o significado de um ou de vários conceitos, para um ou vários indivíduos, em uma ou em várias situações. A experiência empírica tem demonstrado a fidedignidade do diferencial semântico como medida de significado em uma ampla variedade de condições (Pereira, 1986) e também a facilidade de compreensão da tarefa pelos participantes que respondem ao instrumento (Engelmann, 1978).

Bortoloti e de Rose (2007) submeteram estudantes universitários a um treinamento de discriminações condicionais para gerar classes de equivalência envolvendo expressões faciais e figuras abstratas. Em seguida, os participantes avaliaram algumas das figuras abstratas utilizando um instrumento de diferencial semântico formado por um conjunto de 13 escalas ancoradas por adjetivos opostos, cada uma com sete intervalos. Um grupo controle, que não foi ensinado a relacionar os estímulos envolvidos nesse estudo, avaliou as figuras abstratas e as faces utilizando o mesmo instrumento. As figuras foram avaliadas como 
neutras pelo grupo controle (a média das avaliações tendeu ao intervalo central nas diferentes escalas, ao qual foi atribuído o valor zero) e as faces foram avaliadas diferencialmente, conforme a emoção expressada; por exemplo, uma face alegre foi avaliada como positiva e uma raivosa como negativa. Os valores atribuídos às avaliações das faces e dos estímulos equivalentes a elas (feitas pelos participantes do grupo submetido ao treino de relações condicionais) ficaram próximos. As similaridades entre as avaliações das faces pelo grupo controle e das figuras pelo grupo experimental são indicações de que pode haver extensão de significados de "referentes" para "símbolos" em simulações experimentais baseadas no modelo de equivalência de estímulos. Esse resultado promoveu uma validação externa da equivalência como um modelo do significado.

Os resultados desse estudo estabeleceram a ocasião para que se ampliasse o âmbito das investigações envolvendo a combinação do modelo de equivalência de estímulos com o diferencial semântico. Bortoloti e de Rose $(2008,2009,2011)$ têm apurado, por exemplo, que o diferencial semântico permite verificar variações no grau de transferência de significados em função da manipulação de parâmetros experimentais. Os trabalhos envolvendo o diferencial semântico fornecem indicações de que o grau de transferência de significados pode variar sistematicamente de acordo com a apresentação atrasada dos estímulos de comparação em tarefas de emparelhamento ao modelo, com a distância nodal que separa os estímulos relacionados ${ }^{1}$ (Bortoloti \& de Rose, 2009) e com o tempo de apresentação dos "estímulos significativos" (Bortoloti \& de Rose, 2008). O diferencial semântico foi sensível à influência dessas três manipulações que o uso exclusivo dos procedimentos clássicos do modelo de equivalência não seria capaz de captar. O diferencial semântico também pode ser útil para detectar se algumas características emocionais de certos estímulos fazem deles estímulos mais “relacionáveis” do que outros (Bortoloti \& de Rose, 2011).

Não obstante os resultados dessa série de experimentos indicarem que o instrumento de diferencial semântico parece adequado para as finalidades às quais tem sido destinado, a validade psicométrica do instrumento não foi avaliada nos estudos descritos acima. A avaliação da validade de

${ }^{1}$ Distância nodal se refere à quantidade de estímulos mediadores presentes em uma classe de equivalência. Se três conjuntos de estímulos são representados pelas letras A, B e C, a emergência da relação CA após o treino das relações $\mathrm{AB}$ e $\mathrm{BC}$ fornece uma evidência de que os estímulos relacionados dos conjuntos A, B e C são equivalentes. Nesse caso, a ligação entre Ae C é intermediada pelos estímulos do conjunto B. No treino de discriminações condicionais para gerar classes de equivalência, os estímulos que estabelecem a ligação entre aqueles que não foram relacionados diretamente são chamados de nódulos. Quando se considera um treino de relações AB, BC e CD, dois nódulos (B e C) estabelecem a ligação entre A e D. A distância nodal entre A e C é, então, menor do que a distância nodal entre A e D. um instrumento pode ser feita com base na sua estrutura interna, considerando as correlações entre as escalas (itens) que abordam o mesmo construto. Um método bastante utilizado nesse tipo de avaliação é a análise fatorial (Primi, Muniz, \& Nunes, 2009), muito empregada nos estudos de Osgood e colaboradores (e.g., Osgood, 1962; Osgood, May, \& Miron, 1975; Osgood et al., 1957). Osgood propôs as escalas que compõem o diferencial semântico como expressões operacionais de certas dimensões primárias de um "espaço semântico" que pode ser multifatorial (ou multidimensional). O espaço semântico é construído com base nos fatores em que as escalas que abordam o mesmo construto podem ser agrupadas.

O presente estudo teve a finalidade de analisar a validade do diferencial semântico com base na sua estrutura interna e a precisão do instrumento no julgamento dos estímulos que têm sido utilizados em estudos envolvendo transferência de significados entre estímulos equivalentes.

\section{Método}

\section{Participantes}

Foram recrutados para este estudo 196 estudantes de graduação da Universidade Federal de São Carlos (UFSCar), sendo 111 do sexo masculino e 85 do sexo feminino. Os estudantes foram convidados a participar do estudo por meio de contatos verbais em sala de aula e na biblioteca da universidade. Os participantes tinham entre 18 e 27 anos de idade $(M=21,4 ; D P=2,2)$.

\section{Materiais}

A Figura 1 reproduz o instrumento utilizado neste estudo, que é constituído por 13 escalas bipolares de sete intervalos ancoradas, em suas extremidades, à direita e à esquerda, por adjetivos opostos.

$\begin{aligned} \text { TRISTE } & \text { ALEGRE } \\ \text { RELAXADO } & \text { TENSO } \\ \text { ÁSPERO } & \text { LISO } \\ \text { LENTO } & \text { RÁPIDO } \\ \text { BONITO } & \text { FEIO } \\ \text { LEVE } & \text { PESADO } \\ \text { NEGATIVO } & \text { POSITIVO } \\ \text { ATIVO } & \text { PASSIVO } \\ \text { MACIO } & \text { DURO } \\ \text { MAU } & \text { BOM } \\ \text { AGRADÁVEL } & \text { DESAGRADÁVEL } \\ \text { POBRE } & \text { RICO } \\ \text { DOMINANTE } & \text { SUBMISSO }\end{aligned}$

Figura 1. Instrumento de diferencial semântico utilizado neste estudo. 
Almeida, J. H., Bortoloti, R., Ferreira, P. R. S., Schelini, P. W. \& Rose, J. C. C. (2014). Análise das Propriedades Psicométricas de Instrumento de Diferencial Semântico.

Para cada estímulo apresentado, o participante devia assinalar em cada escala um dos intervalos extremos se achasse que o conceito estava "muito fortemente" relacionado com o adjetivo vizinho, o imediatamente mais central se julgasse o relacionamento como do tipo "mais ou menos" e o terceiro de fora para dentro se considerasse que o conceito estava "apenas levemente” relacionado ao adjetivo. $\mathrm{O}$ intervalo central era a origem e o ponto neutro de cada escala; deveria ser assinalado se o conceito estivesse, para o participante, igualmente relacionado aos dois adjetivos ou se considerasse que a escala não tinha qualquer relação com o estímulo. Para efeito de análise dos resultados, foi atribuído o valor 0 (zero) para o intervalo central. Os demais intervalos recebem quantificadores expressos por valores numéricos compreendidos entre -3 e +3. Por exemplo, na escala Bom :_:_:_:_:_:_: Mau, em que o adjetivo “Bom” é considerado positivo e o adjetivo “Mau” é considerado negativo, os valores entre esses dois extremos polares vão de +3 no extremo esquerdo da escala a -3 no extremo direito. Cada valor marca, portanto, a direção do pólo escolhido e a distância em relação a ele. Direção e distância correspondem, respectivamente, à qualidade e à intensidade do significado do conceito julgado na escala bipolar.

Os estímulos utilizados neste estudo foram os mesmos que têm sido utilizados nos trabalhos anteriores: fotografias de faces expressando emoções e figuras abstratas.

Os participantes receberam 14 folhas impressas sobrepostas. As duas primeiras folhas, reproduzidas em anexo, apresentavam as instruções a serem observadas durante a realização da tarefa. Cada uma das 12 folhas seguintes continha impressa em sua parte superior uma fotografia ou uma figura abstrata que o participante deveria avaliar utilizando as 13 escalas bipolares que compunham o instrumento de diferencial semântico impresso logo abaixo da fotografia ou do estímulo abstrato.

\section{Procedimento}

Os participantes receberam instruções (ver Anexo) descrevendo o significado de cada intervalo do instrumento. Todos os participantes avaliaram 12 estímulos (seis fotografias de faces expressando emoções e seis figuras abstratas) utilizando o mesmo instrumento de diferencial semântico. A Figura 2 reproduz os estímulos avaliados na ordem em que foram apresentados aos participantes.

Cada participante avaliou os 12 estímulos utilizando 13 escalas, totalizando 156 avaliações. Os resultados obtidos de todos os participantes serão apresentados a seguir.

\section{Resultados}

Foi realizada uma Análise de Fatores do tipo Análise dos Componentes Principais (ACP). Este tipo de análise permitiu a transformação das variáveis originais em um
2

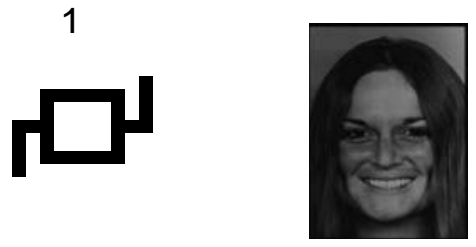

3
4

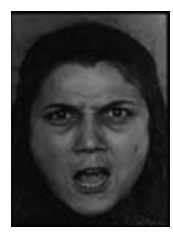

7

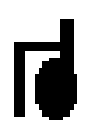

10

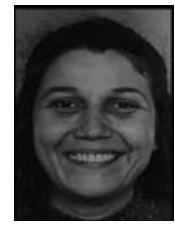

5

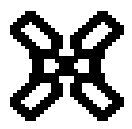

8

11

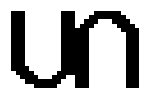

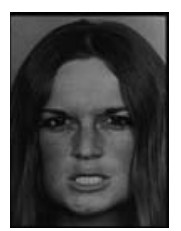

6

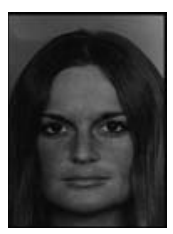

9

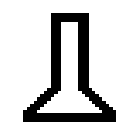

12

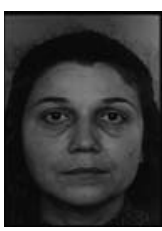

Figura 2. Estímulos avaliados pelos 196 participantes deste estudo. Os números acima de cada figura indicam a ordem de apresentação dos estímulos e não estavam presentes nas folhas entregues aos participantes.

conjunto menor de componentes. O índice Kaiser-Meyer-Olkin (KMO) $)^{2}$ obtido foi de 0,91 , e o teste de esfericidade de Barlett ${ }^{3}$ teve o resultado de 13025,93 $(p<0,001)$, valores considerados aptos para o procedimento fatorial (Pasquali 2005).

Um gráfico de sedimentação sugeriu uma solução com dois fatores como mais adequada, explicando 55,8 \% da variância total dos dados. A Figura 3 mostra o gráfico de sedimentação obtido, com a indicação da melhor quantidade de fatores a serem extraídos. Vale ressaltar que o número de fatores a serem extraídos é indicado acima da linha tracejada que corta o gráfico horizontalmente.

\footnotetext{
${ }^{2} \mathrm{O}$ teste Kaiser-Meyer-Olkin (KMO) indica a proporção da variância dos dados que pode ser considerada comum a todas as variáveis, ou seja, que pode ser atribuída a um fator comum, então: quanto mais próximo de 1 (unidade) melhor o resultado, ou seja, mais adequada é a amostra à aplicação da análise fatorial.

${ }^{3} \mathrm{O}$ teste de esfericidade de Bartlett testa se a matriz de correlação é uma matriz identidade, o que indicaria que não há correlação entre os dados. Procura-se para um nível de significância assumido em 5\% rejeitar a hipótese nula de matriz de correlação identidade.
} 


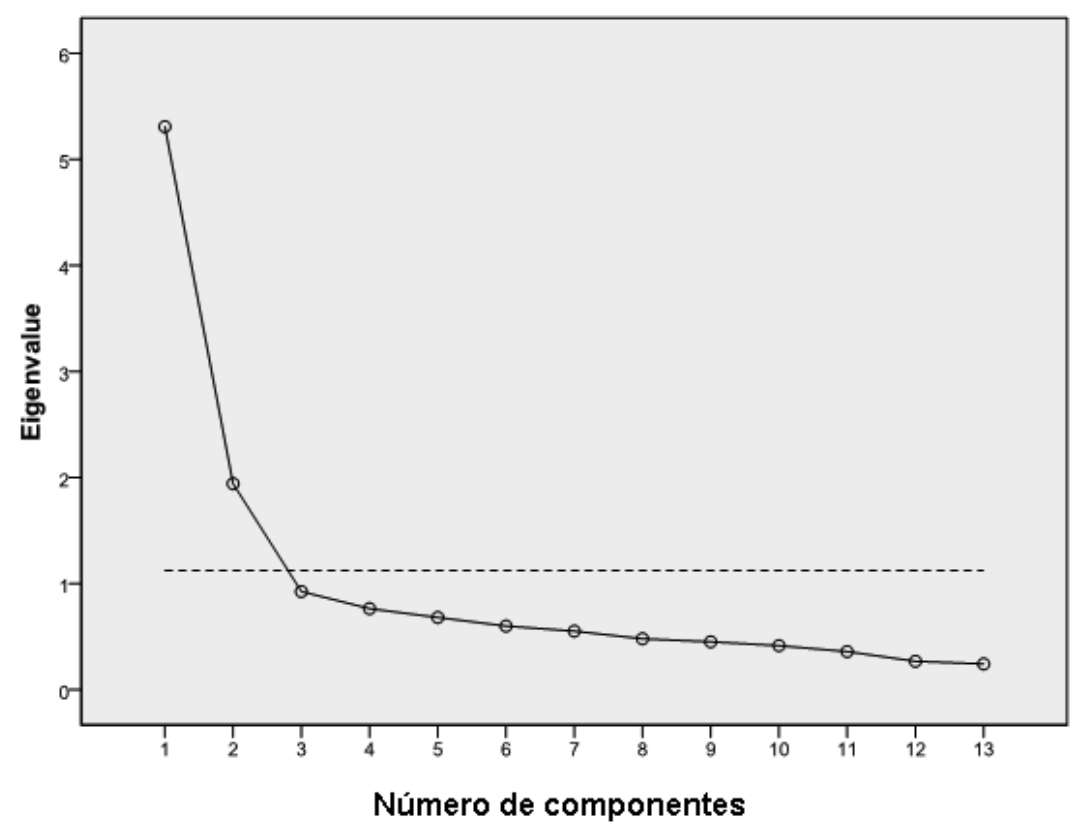

Figura 3. Gráfico de sedimentação gerado pela análise de componentes principais dos dados obtidos.

A extração dos dois fatores sugeridos pelo gráfico de sedimentação foi realizada pelo método de fatoração dos eixos principais (principal axis factoring), com procedimento de rotação do tipo promax. Definiu-se que seriam excluídos da solução fatorial final os itens com cargas fatoriais inferiores a 0,40 (Hair, Anderson, Tatham, \& Black, 1999; Kline, 1994). Com base nesse critério, nenhum dos 13 itens foi excluído. A Tabela 1 apresenta os itens com suas respectivas cargas fatoriais já distribuídos entre os dois fatores.

O Fator 1 obteve um coeficiente alfa, indicativo da precisão, considerado ótimo $(0,91)$ e o Fator 2 obteve um coeficiente alfa moderado $(0,62)$, mas apto a ser utilizado (Nunnally, 1978). O Fator 1 - que parece mensurar, em seu conjunto, características inerentes a qualidades físicas e afetivas dos estímulos avaliados - recebeu o nome de "Avaliação"; o Fator 2 - que parece mensurar características combinadas de atividade e potência dos estímulos avaliados - recebeu o nome de "Potência".

\section{Discussão}

Osgood e colaboradores (e.g., Osgood, 1952, 1962; Osgood et al., 1975; Osgood et al., 1957) postularam que os significados têm múltiplas dimensões que podem ser medidas por meio de escalas apropriadas. O processo de elaboração de escalas sugerido por Osgood e colaboradores para dar conta da multidimensionalidade dos significados envolve a concepção de escalas contínuas, bipolares, ancoradas por adjetivos opostos. Vistas em conjunto, representam uma série de contínuos que vão de um adjetivo ao seu oposto. Alguns desses contínuos devem ser essencialmente equivalentes e, portanto, podem ser representados por
Tabela 1

Solução Fatorial com as Cargas Fatoriais, Comunalidades, Percentual de Variância e Alphas de Cronbach para Todos os Estímulos Avaliados

\begin{tabular}{lccc}
\hline \multirow{2}{*}{ Itens } & \multicolumn{2}{c}{ Fatores } & \multirow{2}{*}{ h2 } \\
\cline { 2 - 3 } & $\mathrm{F} 1$ & $\mathrm{~F} 2$ & \\
\hline Alegre/Triste & 0,70 & & 0,57 \\
Relaxado/Tenso & 0,81 & & 0,65 \\
Liso/Áspero & 0,72 & & 0,51 \\
Bonito/Feio & 0,70 & & 0,53 \\
Leve/Pesado & 0,72 & & 0,53 \\
Positivo/Negativo & 0,86 & & 0,75 \\
Macio/Duro & 0,61 & & 0,41 \\
Bom/Mau & 0,83 & & 0,68 \\
Agradável/Desagradável & 0,85 & & 0,73 \\
Rápido/Lento & & 0,62 & 0,39 \\
Ativo/Passivo & & 0,79 & 0,62 \\
rico/pobre & & 0,42 & 0,25 \\
dominante/submisso & & 0,80 & 0,63 \\
\hline Número de itens & 9 & 4 & \\
\% da variância explicada & 40,83 & 14,97 & \\
Alfa de Cronbach & 0,91 & 0,62 & \\
\hline
\end{tabular}

uma única dimensão. Um número limitado de dimensões pode ser utilizado para definir um espaço semântico dentro do qual os significados dos conceitos de interesse do pesquisador podem ser especificados. O método indicado 
Almeida, J. H., Bortoloti, R., Ferreira, P. R. S., Schelini, P. W. \& Rose, J. C. C. (2014). Análise das Propriedades Psicométricas de Instrumento de Diferencial Semântico.

para a extração das dimensões que irão compor o espaço semântico a partir da série de contínuos elaborados é a análise fatorial, procedimento empregado neste estudo.

As escalas alegre-triste, relaxado-tenso, liso-áspero, bonito-feio, leve-pesado, positivo-negativo, macio-duro, bom-mau e agradável-desagradável apresentaram altos índices de correlação entre si e puderam ser representadas como uma das dimensões do espaço semântico, que recebeu o nome de “Avaliação”. Os índices de correlação obtidos para as escalas rápido-lento, ativo-passivo, rico-pobre e dominante-submisso não foram tão elevados, mas permitiram a representação de uma segunda dimensão, ainda que provisória, desse mesmo espaço semântico, recebendo o nome de "Potência”. Os nomes “Avaliação" e "Potência” foram escolhidos em referência às dimensões mais relevantes do significado apontadas em diversos estudos de Osgood e colaboradores.

A possibilidade de agrupar diversas escalas em fatores ou, no caso deste estudo, em dimensões do espaço semântico, é o que torna possível a elaboração de um instrumento de medida quantitativa do significado. Se as escalas utilizadas fossem independentes umas das outras, as medições quantitativas teriam sérias limitações e as comparações entre os significados dos conceitos de interesse seriam muito mais difíceis de serem estabelecidas. Efetuada a análise fatorial, o significado que um estímulo tem para um participante ou grupo de participantes e a distância que diversos estímulos mantêm entre si podem ser verificados com base nas dimensões do espaço semântico. Para efetuar essas análises, os sete segmentos das escalas recebem valores numéricos que vão $-3 \mathrm{a}+3$, conforme mencionado anteriormente. Para encontrar o lugar de cada estímulo no espaço semântico, deve-se obter o escore desse estímulo em cada um dos fatores. Esse escore é obtido pela soma dos escores de cada escala de um fator dividida pelo número de escalas nesse fator. Para efeito de exemplo, a Figura 4 ilustra uma forma de representar graficamente as duas dimensões do espaço semântico que é possível criar a partir dos dois fatores extraídos das 13 escalas que compõem o diferencial semântico utilizado neste estudo. Neste exemplo, é possível estimar a distância entre os significados atribuídos, pelos 196 participantes deste estudo, a uma mesma face quando ela expressa alegria, raiva e neutralidade.

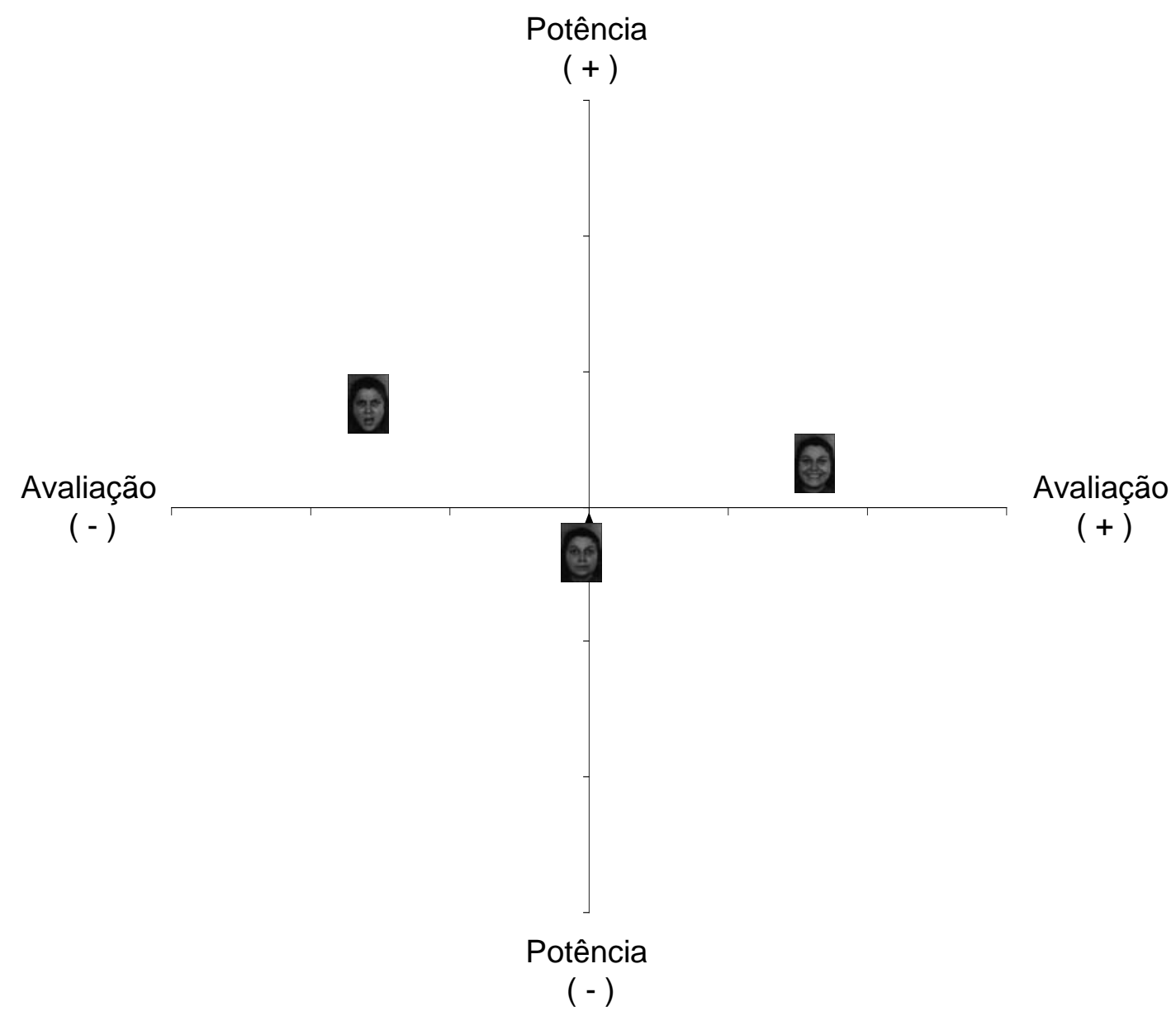

Figura 4. Distribuição do significado de três expressões faciais pelo espaço semântico criado a partir dos dois fatores extraídos do instrumento. 
A face alegre foi avaliada de forma positiva e a raivosa de forma negativa, mas a face raivosa foi classificada como mais potente do que a face alegre. Já a face neutra, recebeu avaliações neutras e foi classificada como a menos potente dentre as emoções que os participantes foram instruídos a julgar.

Este estudo pode ser entendido como uma primeira tentativa de validação de um instrumento de medida de transferência de significados entre estímulos equivalentes. Apesar de a análise fatorial ter indicado que o instrumento parece ter uma estrutura interna válida, algumas considerações quanto à precisão (fidedignidade) do instrumento precisam ser destacadas. A precisão pode ser entendida como a consistência ou estabilidade de uma medida do comportamento (Anastasi \& Urbina, 2000). Um instrumento é considerado fidedigno quando proporciona uma medida estável da variável, ou seja, uma medida que não "flutua" significativamente entre uma aplicação e outra. Um dos indicadores de estabilidade mais utilizados é o coeficiente alfa, em que se calcula a correlação de cada item com todos os demais, indicando uma correlação média entre as variáveis (Anastasi \& Urbina, 2000).

Conforme apresentado na seção anterior, um coeficiente alfa de 0,62 para o segundo fator (que deu origem à dimensão Potência) é considerado baixo, mas, para alguns autores (e.g., Nunnally, 1978), ainda aceitável. Murphy e Davidshofer (1998) consideram inaceitáveis valores de alfa abaixo de 0,6. Ainda que tenha ultrapassado o limiar daquilo que os autores citados consideram como "aceitável”, o coeficiente obtido para o segundo fator indica que ele pode apresentar um erro de medida muito mais elevado do que o primeiro fator (Avaliação, que apresentou um coeficiente alfa considerado ótimo, de 0,91 ). O segundo fator deve, portanto, ser revisto para que se torne mais adequado e relevante para a estrutura do instrumento apresentado neste artigo.

$\mathrm{O}$ modelo de equivalência de estímulos, entendido como um modelo experimental de relações simbólicas, pode se beneficiar de instrumentos capazes de medir a transferência de significados entre estímulos equivalentes. Bortoloti e de Rose têm encontrado evidências de que a combinação do emparelhamento ao modelo com o diferencial semântico permite investigar parâmetros experimentais que determinam diferenças quantitativas entre as relações de equivalência estabelecidas (Bortoloti \& de Rose, 2008, 2009, 2011). Essa investigação dificilmente poderia ser feita com a utilização da metodologia especificada pelo modelo de equivalência, baseada em procedimentos de emparelhamento ao modelo. A razão disso é que procedimentos de emparelhamento ao modelo estabelecem contingências de escolhas forçadas entre alternativas discretas que podem determinar se o participante formou ou não formou classes de estímulos equivalentes (efeito “tudo ou nada” ou escala binária zero ou um), mas é muito difícil determinar com precisão, por exemplo, se os estímulos presentes nas classes formadas estão igualmente relacionados entre si. Em outros termos, o padrão meto- dológico utilizado em pesquisas envolvendo o modelo de equivalência permite que se constate a existência de uma relação simbólica, mas não que se avaliem diferentes graus de relacionamento.

Uma estimativa de diferenças quantitativas entre relações de equivalência pode ser feita pela mensuração do nível de transferência de funções nas classes estabelecidas. Variações na transferência de funções indicam que as classes de equivalência podem conter estímulos mais ou menos relacionados entre si, ou seja, indicam variações no grau de relacionamento de estímulos equivalentes (cf. Fields, Adams, Verhave, \& Newman, 1993). Se uma dada operação que é ocasionada em um determinado nível por um dos membros de uma classe de equivalência passa a ser ocasionada em níveis similares pelos demais membros da classe, então todos esses estímulos poderiam estar igualmente relacionados. Se, por outro lado, essa operação passa a ser ocasionada em níveis diferentes, então os estímulos não estariam igualmente relacionados. Nesse segundo caso, quando o grau de transferência varia sistematicamente de acordo com os parâmetros experimentais envolvidos, as variações no grau de relacionamento detectadas poderiam ser atribuídas aos parâmetros que foram utilizados. O instrumento de diferencial semântico avaliado tem se mostrado sensível a manipulações paramétricas sobre a transferência de significados entre estímulos equivalentes. Os resultados deste estudo indicam que uma revisão do instrumento é necessária (especialmente uma revisão do segundo fator), mas que vale a pena continuar investindo neste instrumento.

\section{Referências}

Anastasi, A., \& Urbina, S. (2000). Testagem psicológica (7. ed.). Porto Alegre, RS: Artes Médicas.

Bortoloti, R., \& de Rose, J. C. (2007). Medida do grau de relacionamento entre estímulos equivalentes. Psicologia: Reflexão e Crítica, 20, 252-258.

Bortoloti, R., \& de Rose, J. C. (2008). Transferência de significado de expressões faciais apresentadas brevemente para estímulos abstratos equivalentes a elas. Acta Comportamentalia, 16(2), 223-241.

Bortoloti, R., \& de Rose, J. C. (2009). Assessment of the relatedness of equivalent stimuli through a semantic differential. The Psychological Record, 59, 563-590.

Bortoloti, R., \& de Rose, J. C. (2011). Avaliação do efeito de dica semântica e da indução de significado entre estímulos abstratos equivalentes. Psicologia: Reflexão e Crítica, 24(2), 381-393.

Engelmann, A. (1978). Os estados subjetivos, uma tentativa de classificação de seus relatos verbais. São Paulo, SP: Ática.

Fields, L., Adams, B. J., Verhave, T., \& Newman, S. (1993). Are stimuli in equivalence classes equally related to each other? The Psychological Record, 43, 85-105.

Hair, J. F., Jr., Anderson, R. E., Tatham, R. L., \& Black, W. C. (1999). Análisis multivariante. Madrid, España: Prentice Hall International.

Kline, P. (1994). An easy guide to factor analysis. New York: Routlege. 
Almeida, J. H., Bortoloti, R., Ferreira, P. R. S., Schelini, P. W. \& Rose, J. C. C. (2014). Análise das Propriedades Psicométricas de Instrumento de Diferencial Semântico.

Murphy, K. R., \& Davidshofer, C. O. (1998). Psychological testing: Principles and applications. Upper Saddle River, NJ: Prentice-Hall.

Nunnally, J. C. (1978). Psychometric theory. New York: McGraw-Hill.

Osgood, C. E. (1952). The nature and measurement of meaning. Psychological Bulletin, 49, 197-237.

Osgood, C. E. (1962). Studies on the generality of meaning systems. American Psychologist, 17, 10-28.

Osgood, C. E., May, W. H., \& Miron, M. S. (1975). Cross-cultural universals of affective meaning. Urbana, IL: University of Illinois Press.

Osgood, C. E., Suci, G. I., \& Tannenbaum, P. H. (1957). The measurement of meaning. Urbana, IL: University of Illinois Press.

Pasquali, L. (2005). Extração dos fatores. In L. Pasquali (Ed.), Análise fatorial para pesquisadores (pp. 55-86). Brasília, DF: Laboratório de Pesquisa em Avaliação e Medida, Universidade de Brasília.

Pereira, C. A. A. (1986). O diferencial semântico: Uma técnica de medida nas ciências humanas e sociais. São Paulo, SP: Ática.

Primi, R., Muniz, M., \& Nunes, C. H. S. S. (2009). Definições contemporâneas de validade de testes psicológicos. In C. S. Hutz (Ed.), Avanços e polêmicas em avaliação psicológica (pp. 243-265). São Paulo, SP: Casa do Psicólogo.

Sidman, M. (1971). Reading and auditory-visual equivalences. Journal of Speech and Hearing Research, 14, 5-13.

Sidman, M. (1986). Functional analysis of emergent verbal classes. In T. Thompson \& M. D. Zeiler (Eds.), Analysis and integration of behavioral units. Hillsdale, NJ: Lawrence Erlbaum.

Sidman, M. (1990) Equivalence relations: Where do they come from? In D. E. Blackman \& H. Lejeune (Eds.), Behavior analysis in theory and practice: Contributions and controversies. Hillsdale, NJ: Lawrence Erlbaum.

Sidman, M. (1994). Equivalence relations and behavior. A research story. Boston, MA: Authors Cooperative.

Sidman, M., \& Cresson, O. (1973). Reading and crossmodal transfer of stimulus equivalences in severe retardation. American Journal of Mental Deficiency, 77, 515-523.

Sidman, M., \& Tailby, W. (1982). Conditional discrimination VS. Matching to sample: An expansion of the testing paradigm. Journal of the Experimental Analysis of Behavior, 37, 5-22. 


\section{Anexo}

Nome (ouiniciais):

Data de nascimento: Data da aplicação:

ocupação atual (se estudante, especificar o curso):

\section{INSTRUÇÕES:}

Você encontrará desenhos e faces de pessoas no alto de cada uma das páginas seguintes. Abaixo de cada figura e face haverá pares de adjetivos, sendo um adjetivo de sentido oposto ao outro. Faça um “X” no espaço mais próximo do adjetivo que achar que melhor representa a figura ou a face. Preste atenção no exemplo abaixo. Observe primeiramente o desenho.

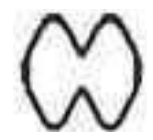

Este desenho pode, de maneira geral, ser entendido como bonito ou feio.

Se você achar que o desenho acima é extremamente bonito, terá que colocar o X no espaço mais próximo do adjetivo bonito. Assim:

\begin{tabular}{|l|l|l|l|l|l|l|l|l|}
\hline BONITO & $\mathbf{X}$ & & & & & & & FEIO \\
\hline
\end{tabular}

Se achar que é extremamente feio, terá que colocar o X na outra ponta, no espaço mais próximo de feio. Assim:

\begin{tabular}{|l|l|l|l|l|l|l|l|l|}
\hline BONITO & & & & & & & $\mathbf{X}$ & FEIO \\
\hline
\end{tabular}

Se achar o desenho regularmente bonito, deverá por o X no segundo espaço mais próximo de bonito. Assim:

\begin{tabular}{|l|l|l|l|l|l|l|l|l|}
\hline BONITO & & $\mathbf{X}$ & & & & & & FEIO \\
\hline
\end{tabular}

Se julgá-lo regularmente feio, deverá por o X no segundo espaço mais próximo de feio. Assim:

\begin{tabular}{|l|l|l|l|l|l|l|l|l|}
\hline BONITO & & & & & & $\mathbf{X}$ & & FEIO \\
\hline
\end{tabular}

Se for considerado por você levemente bonito, deverá colocar o X no terceiro espaço a partir de bonito. Assim:

\begin{tabular}{|l|l|l|l|l|l|l|l|l|}
\hline BONITO & & & $\mathbf{X}$ & & & & & FEIO \\
\hline
\end{tabular}

E se julgá-lo levemente feio, o X deverá aparecer no terceiro espaço a partir de feio. Assim:

\begin{tabular}{|l|l|l|l|l|l|l|l|l|}
\hline BONITO & & & & & $\mathbf{X}$ & & & FEIO \\
\hline
\end{tabular}

Caso você ache que a figura impressa no alto da folha não tem nada a ver com aquele par de adjetivos ou tem relação tanto com um quanto com outro, ponha o X no espaço do meio (abaixo do “.”). Assim:

\begin{tabular}{|l|l|l|l|l|l|l|l|l|}
\hline BONITO & & & & $\mathbf{X}$ & & & & FEIO \\
\hline
\end{tabular}


Almeida, J. H., Bortoloti, R., Ferreira, P. R. S., Schelini, P. W. \& Rose, J. C. C. (2014). Análise das Propriedades Psicométricas de Instrumento de Diferencial Semântico.

Agora, faça um exemplo na próxima página sozinho(a), olhando para face e assinalando os espaços.
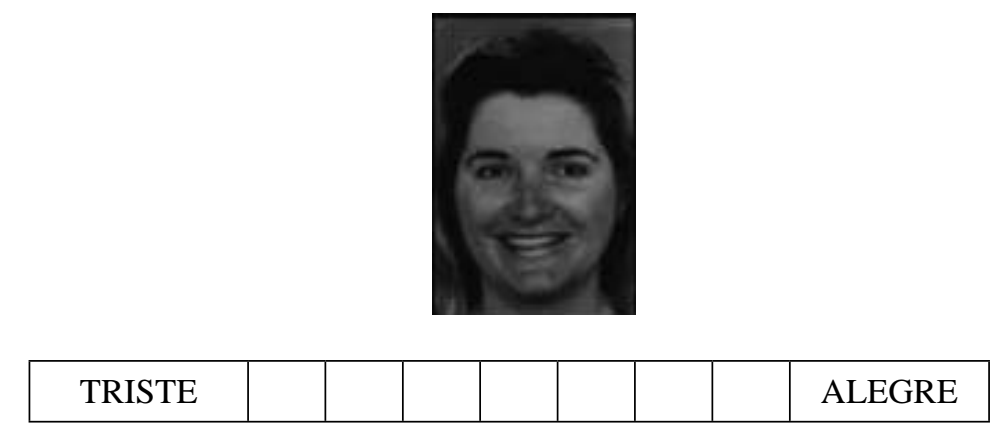

\begin{tabular}{|l|l|l|l|l|l|l|l|l|}
\hline RELAXADO & & & & & & & & TENSO \\
\hline
\end{tabular}

\begin{tabular}{|l|l|l|l|l|l|l|l|l|}
\hline ÁSPERO & & & & & & & & LISO \\
\hline
\end{tabular}

\begin{tabular}{|l|l|l|l|l|l|l|l|l|}
\hline LENTO & & & & & & & & RÁPIDO \\
\hline
\end{tabular}

\begin{tabular}{|l|l|l|l|l|l|l|l|l|}
\hline BONITO & & & & & & & & FEIO \\
\hline
\end{tabular}

\begin{tabular}{|c|c|c|c|c|c|c|c|c|}
\hline LEVE & & & & & & & & PESADO \\
\hline
\end{tabular}

\begin{tabular}{|c|c|c|c|c|c|c|c|c|}
\hline NEGATIVO & & & & & & & & POSITIVO \\
\hline
\end{tabular}

\begin{tabular}{|l|l|l|l|l|l|l|l|l|}
\hline ATIVO & & & & & & & & PASSIVO \\
\hline
\end{tabular}

\begin{tabular}{|l|l|l|l|l|l|l|l|l|}
\hline MACIO & & & & & & & & DURO \\
\hline
\end{tabular}

\begin{tabular}{|l|l|l|l|l|l|l|l|l|}
\hline MAU & & & & & & & & BOM \\
\hline
\end{tabular}

\begin{tabular}{|l|l|l|l|l|l|l|l|l|}
\hline AGRADÁVEL & & & & & & & & DESAGRADÁVEL \\
\hline
\end{tabular}

\begin{tabular}{|l|l|l|l|l|l|l|l|l|}
\hline POBRE & & & & & & & & RICO \\
\hline
\end{tabular}

\begin{tabular}{|l|l|l|l|l|l|l|l|l|}
\hline DOMINANTE & & & & & & & & TENSO \\
\hline
\end{tabular}

Continue fazendo a atividade, mas preste atenção porque terá que olhar para cada figura ou face e assinalar todos os pares de adjetivos. Você encontrará 13 pares de adjetivos abaixo de cada figura ou face e, portanto, deverá assinalar 13 espaços. Não deixe nenhum par de adjetivos sem assinalar.

Não se preocupe em acertar ou errar, porque não há uma resposta certa ou errada. Procure ser sincero.

Por favor, não deixe qualquer desenho ou face sem assinalar.

Se você tiver alguma dúvida depois de ter lido as instruções, chame a pessoa que estiver aplicando este questionário e faça-lhe perguntas sobre o que não entendeu.

Obrigado pela sua colaboração. 